\title{
RENDICIÓN DE CUENTAS EN EL XV ANIVERSARIO DE CREACIÓN DE LA UNIVERSIDAD ESTATAL PENÍNSULA DE SANTA ELENA
}

\section{INTRODUCCIÓN}

Estamos culminando el tercer año de la actual administración. Hemos comenzado a transitar el cuarto año de nuestro periodo, el mismo que concluirá el mes de mayo del 2015.

Cabe recordar que con fecha 4 de noviembre del 2009, seis meses antes de ser electo, el entonces CONEA, presentó el denominado Informe de Evaluación de Desempeño Institucional de las Universidades y Escuelas Politécnicas del Ecuador. El mencionado Informe, ubicó a la UPSE en la categoría E.

Una de las recomendaciones, donde mayor énfasis se ponía, era en la Planificación Estratégica. La recomendación tenía y tiene hoy una enorme carga de sentido común, puesto que la improvisación y la intuición no son los mejores instrumentos para alcanzar metas y/o lograr propósitos.

La UPSE contaba con un Plan Estratégico de Desarrollo Institucional 2009-2013 (PEDI), documento que se encontraba sin ejecución desde el 28 de Febrero del 2009, fecha en que fue aprobado.

El reajuste del PEDI fue una de nuestras primeras tareas. Las modificaciones se iniciaron en agosto del 2010 y fueron aprobadas el 27 de diciembre del 2010.

Emprendíamos en la tarea de construir una Cultura de Planificación en todos los estamentos de la Universidad. Debíamos orientar a la UPSE hacia el logro de su Misión y para ello recurríamos a los presupuestos técnicos de la Planificación Estratégica.

Al finalizar el presente año 2013 habremos concluido el actual PEDI.

\section{1.- PLAN ESTRATÉGICO DE DESARROLLO INSTITUCIONAL (PEDI 2009 2013)}

El índice global de cumplimiento del PEDI (2009-2013) es del 70,72\%, faltando aún seis meses por concluir. Se puede colegir que aumentaremos el índice de desempeño, puesto que aún falta por evaluar lo que resta del presente año.

CUMPLIMIENTO DEL PEDI 2009-2013 POR FUNCIÓN

\begin{tabular}{|c|c|c|}
\hline FUNCIÓN & $\begin{array}{c}\text { CUMPLIMIENTO } \\
\text { PEDI 2009-2013 }\end{array}$ & $\begin{array}{l}\text { \% NO CUMPLIMIENTO- } \\
\text { PEDI 2009-2013 }\end{array}$ \\
\hline DOCENCIA & $63,00 \%$ & $37,00 \%$ \\
\hline INVESTIGACIÓN & $71,19 \%$ & $28,81 \%$ \\
\hline GESTIÓN ADMINISTRATIVA & $73,28 \%$ & $26,72 \%$ \\
\hline VINCULACIÓN & $75,00 \%$ & $25,00 \%$ \\
\hline
\end{tabular}

\section{FORTALEZAS}

Las fortalezas que hemos alcanzado en la ejecución del presente PEDI son:

a. $58 \%$ de las carreras han mejorado sus diseños curriculares

b. $77 \%$ de las carreras cuentan con estudios de necesidades e investigación de mercado

c. $66 \%$ de los docentes han sido capacitados en docencia, investigación y vinculación con la colectividad.

d. $61 \%$ de docentes tienen título de maestría

e. 41 profesionales que laboran en el ámbito administrativo tienen título de maestría

f. $21 \%$ de los convenios suscritos están relacionados con ciencia, tecnología e innovación

g. Se han ejecutado 64 eventos científicos, simposios, congresos y actividades que han fomentado, estimulado y desarrollado la práctica de la ciencia en nuestra provincia. 
h. $94 \%$ de trabajadores evaluados

i. Existencia de un estudio de necesidades institucionales para la ejecución de capacitaciones de los trabajadores.

j. 91\% de trabajadores capacitados de acuerdo a necesidades institucionales

k. Se han otorgado 38 becas para estudios de cuarto nivel.

I. La UPSE tiene sistematizado el $60 \%$ de sus procesos de información.

m. 71\% de cumplimiento de metas del PEDI.

$\mathrm{n}$. Se ha superado la meta de contar con 5 profesores con título de Ph.D. Actualmente trabajan en nuestra institución 7 docentes con este tipo de formación académica.

\section{2.- PLAN OPERATIVO ANUAL (POA) 2012}

En el POA 2012, el logro de las metas de cada indicador de éxito ha alcanzado el nivel de logro institucional del $80 \%$, tal como se muestra en la siguiente tabla:

\section{AVANCE DEL POA 2012 POR UNIDADES ACADÉMICAS Y DE GESTIÓN}

\begin{tabular}{|c|c|}
\hline UNIDADES & $\begin{array}{r}\text { PORCENTAJE DE } \\
\text { AVANCE }\end{array}$ \\
\hline ACADEMIA & $68,03 \%$ \\
\hline GESTIÓN & $91,23 \%$ \\
\hline
\end{tabular}

CUMPLIMIENTO DEL POA 2012 POR FUNCIÓN

\begin{tabular}{|c|c|c|}
\hline FUNCIÓN & $\begin{array}{c}\% \text { CUMPLI- } \\
\text { M I E N T O } \\
\text { POA } 2012\end{array}$ & $\begin{array}{l}\% \text { NO CUM- } \\
\text { PLIMIENTO } \\
\text { POA } 2012\end{array}$ \\
\hline DOCENCIA & $79 \%$ & $21 \%$ \\
\hline INVESTIGACIÓN & $74 \%$ & $26 \%$ \\
\hline $\begin{array}{l}\text { GESTIÓN ADMI- } \\
\text { NISTRATIVA }\end{array}$ & $85 \%$ & $15 \%$ \\
\hline VINCULACIÓN & $83 \%$ & $17 \%$ \\
\hline
\end{tabular}

Del POA 2013 nos corresponde rendir cuentas del primer cuatrimestre. El consolidado por funciones muestra:

\section{CUMPLIMIENTO DEL POA 2013 POR FUNCIÓN PRIMER CUATRIMESTRE}

\begin{tabular}{|l|l|l|}
$N^{\circ}$ & FUNCIÓN & $\begin{array}{l}\text { P r o m e d i o } \\
\text { avance por } \\
\text { función }\end{array}$ \\
\hline 1 & DOCENCIA & $74 \%$ \\
\hline 2 & INVESTIGACIÓN & $88 \%$ \\
\hline 3 & $\begin{array}{l}\text { GESTIÓN ADMINISTRA- } \\
\text { TIVA }\end{array}$ & $67 \%$ \\
\hline 4 & VINCULACIÓN & $33 \%$ \\
\hline
\end{tabular}

Como parte de esta rendición de cuentas del periodo julio 2012 a julio 2013, debemos también destacar:

1. HOTEL SAMARINA: Con fecha 11 de octubre del 2012, se firmó la escritura de compra-venta que otorgó el Ministerio de Turismo a favor de la Universidad Estatal Península de Elena, con la cual se legalizó la propiedad del Hotel Samarina, efectuándose posteriormente la inscripción en el Registro de la Propiedad del Cantón La Libertad.

2. SANTA ELENA EP: Se creó la Empresa Pública de Bienes, Obras y Servicios Santa Elena $E P$, con el objetivo de proponer alternativas de solución a las diversas necesidades del país mediante el desarrollo y ejecución de proyectos en distintas áreas del saber, energía, ambiental, recursos renovables y procesos tecnológicos.

3. ALCANTARILLADO SANITARIO Y PLUVIAL: El sistema de alcantarillado sanitario y pluvial del campus de la UPSE, está en funcionamiento y vamos a concluirlo una vez que se terminen de ejecutar las garantías de fiel cumplimiento y buen uso de los anticipos.

4. INCREMENTO DEL PRESUPUESTO 2013: El pasado 29 de abril el Consejo de Educación Superior aprobó la nueva fórmula de distribución de recursos, en base a la calidad de la educación. Con el nuevo método de distribución la UPSE incrementará su presupuesto 2013 en \$1'083.463,54. Una vez que se concrete este incremento, el mismo será destinado para mejoras, privilegiando la inversión en la conectividad y oficinas para los docentes de tiempo completo.

5. INCREMENTO DEL PLAN ANUAL DE INVERSIÓN 2014: El Plan Anual de Inversiones para el 2014, se incrementará de la siguiente forma: 


\begin{tabular}{|l|l|}
\hline \multicolumn{2}{|l|}{ PROYECTO } \\
$\begin{array}{l}\text { EDIFICIO DE GESTIÓN ACADÉ- } \\
\text { MICAY ADMINISTRATIVA }\end{array}$ & $\$ 1.720 .648,20$ \\
\hline COLISEO DE LA UPSE & $\$ 2.763 .668,95$ \\
\hline $\begin{array}{l}\text { BIBLIOTECA GENERAL CON } \\
\text { ATENCIÓN }\end{array}$ & $\$ 579.674,51$ \\
\hline $\begin{array}{l}\text { BLOQUE 4 PLANTAS PARA ESTU- } \\
\text { DIANTES DE POSTGRADO }\end{array}$ & $\$ 1.432 .530,76$ \\
\hline TOTAL & $\$ 6.496 .522,42$ \\
\hline
\end{tabular}

\section{3.- ACREDITACIÓN (enero - mayo 2013)}

El sistema de acreditación que la Constitución de 1998 aprobó, constituyendo el CONEA, era un buen sistema, con aceptable consenso y legitimación, pero su fuerte sesgo corporativo y autónomo, le imposibilitaba impulsar transformaciones significativas y garantizar la calidad.

La nueva Constitución del 2008 introdujo profundas reformas en el sistema de acreditación. Partió del principio que la calidad no se alcanza, si los procesos de acreditación son llevados a cabo por las propias universidades.

Organismos independientes del sistema y sistemas de evaluación sobre indicadores no subjetivos, interpretables por las propias universidades, son los elementos del nuevo proceso. Una de las graves deficiencias, ser juez y parte, fue corregida.

En los primeros meses del presente año del 2013, el CEAACES, sustituto del extinto CONEA, procedió a concretar lo señalado en la transitoria vigésima de la Constitución de la República en la que, se daba un plazo de cinco años para que las instituciones de educación superior, sus carreras y programas sean acreditados.

El proceso de acreditación incluyó proporcionar información al SNIESE, la misma que fue levantada a la plataforma informática de dicho organismo.

Luego, se efectuó la Autoevaluación respectiva y se concluyó con la evaluación externa que fue llevada a cabo por un equipo de pares académicos expertos.

Por tercera ocasión la UPSE volvió a ser evaluada. En esta ocasión con fines de acreditación institucional.

Los indicadores de calidad, del modelo proporcionado por el CEAACES, fueron incorporados, en su oportunidad, al Plan Estratégico Institucional. Esta articulación de: indicadores de calidad y plan estratégico, fueron efectivos instrumentos para enfrentar el proceso de acreditación en el presente año.

\section{4.- AUTOEVALUACIÓN INSTITU- CIONAL}

Como parte de la segunda fase del proceso de Acreditación se realizó la Autoevaluación Institucional; de manera formal, desde el 20 de marzo hasta el 12 de abril del presente año.

De conformidad con el modelo de evaluación del CEAACES, se aplicaron instrumentos de verificación de los documentos que nos permitieran evidenciar el funcionamiento de cada una de las dependencias y poder fundamentar el proceso de toma de decisiones con información relevante y oportuna.

Para desarrollar la autoevaluación recurrimos al modelo socializado por el CEAACES, mismo que está distribuido en 5 criterios.

Los resultados de nuestra Autoevaluación alcanzaron un $70 \%$ de la ponderación total, poniendo en evidencia el progreso obtenido fruto de la constancia y la perseverancia en nuestro compromiso.

\begin{tabular}{|l|l|l|}
\hline \multicolumn{1}{|l}{ CRITERIO } & \multicolumn{1}{l}{$\begin{array}{l}\text { PONDERACION } \\
\text { MODELO }\end{array}$} & $\begin{array}{l}\text { UTILIDAD } \\
\text { UPSE }\end{array}$ \\
\hline ACADEMIA & 40.00 & 33.59 \\
\hline EFICIENCIA ACADÉMICA & 10.00 & 6.70 \\
\hline INFRAESTRUCTURA & 20.00 & 10.65 \\
\hline INVESTIGACIÓN & 10.00 & 4.00 \\
\hline ORGANIZACIÓN & 20.00 & 15.54 \\
\hline TOTAL GENERAL & 100.00 & 70.4 \\
\hline
\end{tabular}

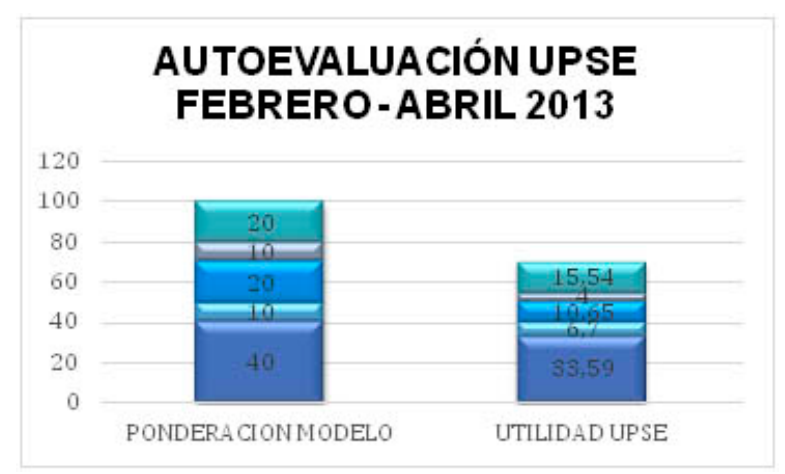

Del criterio Academia merecen destacarse los indicadores siguientes: 
INDICADOR.- DOCTORES TC

En el 2010 no teníamos docentes Ph.D.; para el 2011 se contrataron profesores con título de $\mathrm{PhD}$ $(0,8 \%)$ y en el 2012 a junio del 2013 con la aplicación de los Planes de Mejoras se logró el 4,27\% en este indicador.

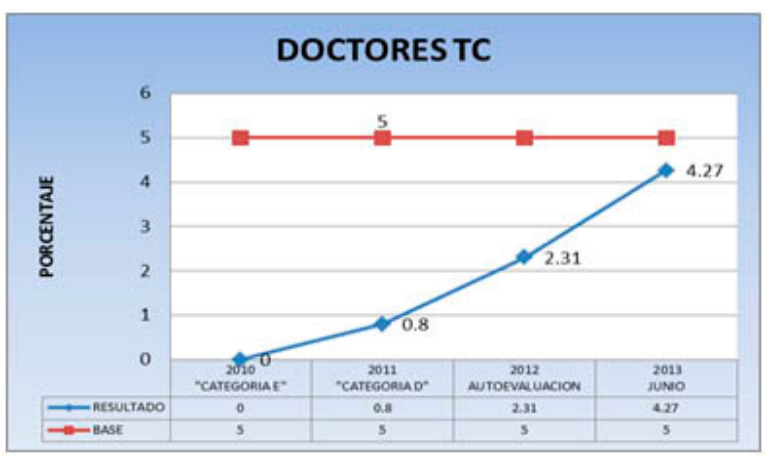

\section{INDICADOR.- DIRECCIÓN MUJERES}

En consideración al principio de igualdad en el cual se manifiesta que tanto hombres como mujeres tienen derecho a las mismas oportunidades, la universidad en la evaluación cuando fue categorizada $\mathrm{E}$ no tenía ninguna mujer, dentro de los cargos de dirección académica, partiendo de ese resultado en el 2011 se logró alcanzar el 23,10\% de mujeres en cargos de dirección académica y del 2012 a junio del 2013 se logró el 27,01\% en dicho indicador.

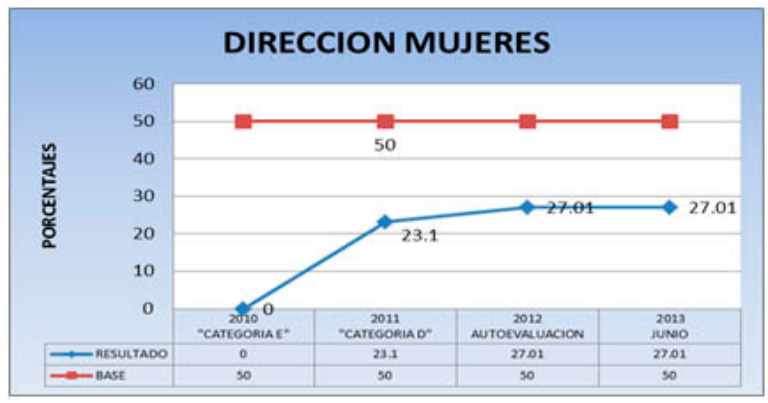

INDICADOR.- DOCENCIA MUJERES

Este indicador muestra un ascenso muy significativo, en cuanto a la inclusión de mujeres dentro de la planta docente, tal como indica el cuadro en el 2011 el 35,1\% y en el 2012 a junio del 2013 el $40,33 \%$, valor cercano a la máxima utilidad que es del $50 \%$ de mujeres del total de la planta docente.

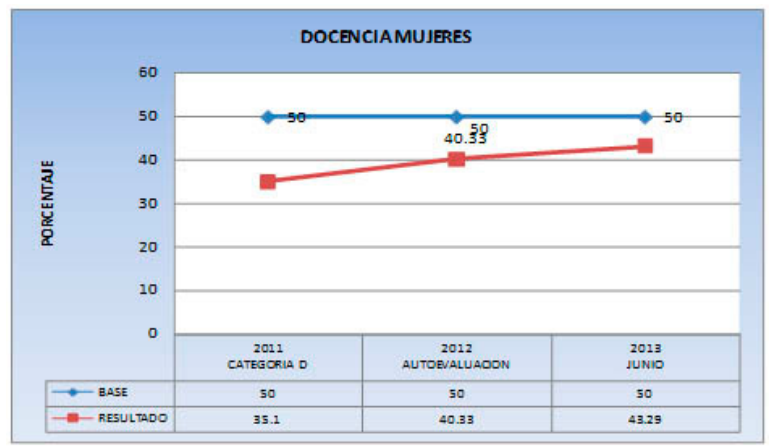

INDICADOR.- ESTUDIANTES POR DOCENTES

TC

Se consideró un valor máximo de 15 estudiantes por cada docente TC. En el año 2010 teníamos 152 estudiantes por docentes TC, con la aplicación de los Planes de Mejoras anuales se realizaron los correctivos y en el año 2011 se obtuvo como promedio 67 estudiantes por cada docentes TC, y para el 2012 a junio del 2013 el resultado obtenido es 20, 07 estudiantes por cada docente TC.

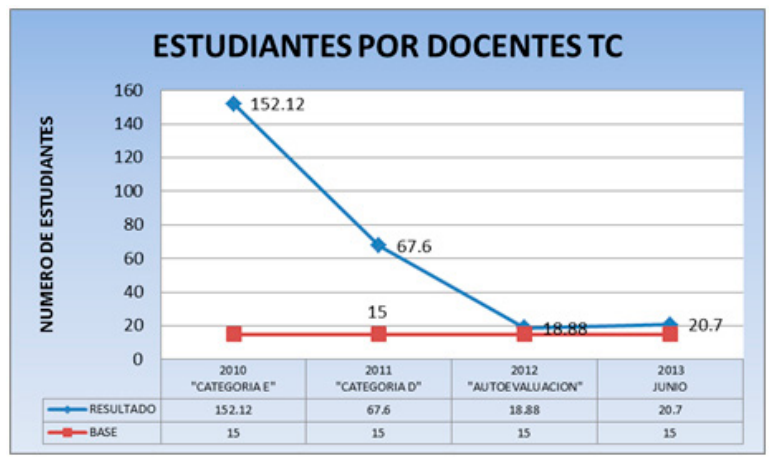

\section{INDICADOR.- EVALUACIÓN A DOCENTES}

En el 2010 no se contaba con un reglamento o normativa que regule y evalúe las actividades asignadas a cada uno de los docentes, con ese antecedente se elaboró y aprobó el reglamento de evaluación de los docentes, el mismo que hoy se aplica en su totalidad.

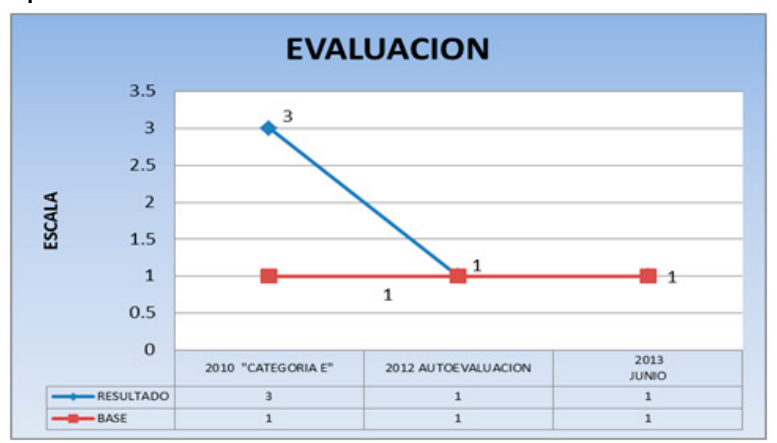

INDICADOR.- HORAS CLASES TC

En el año 2010 se promedió 11 horas de clases de los docentes tiempo completo, en el 2011: 16,6 horas; en el 2012 13,76, y a Junio del 2013: 13,12 horas clases efectivas. En este indicador se cumple con los estándares de calidad establecidos por el CEAACES.

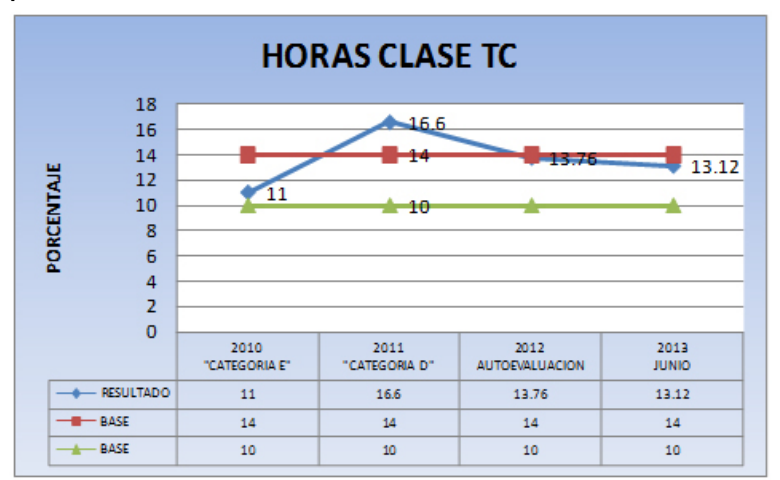


INDICADOR.- DOCENTES TIEMPO COMPLETO

En el 2010 se evidenció el 12,32\% de docentes a tiempo completo, en el 2011: $16,7 \%$ y en el 2012 : $48,08 \%$ de docentes a tiempo completo del total de docentes que prestaron sus servicios profesionales en el año. Se puede evidenciar en el gráfico el esfuerzo por alcanzar el estándar de calidad. De diciembre del 2012 a junio del 2013 se alcanza un promedio superior al 70,99\%. Con lo cual cumplimos con holgura el mandato de la LOES en su décimo cuarta disposicion transitoria.

\section{INDICADOR.- FORMACIÓN POSGRADO}

En la evaluación del 2010 se evidenció el 0,54 semestres de formación de posgrado, en el año 2011: 1,17, semestres de formación y en el 2012 en la autoevaluación a Junio del 2013, se tiene 2,6 semestres, considerando una utilidad máxima de 2,9 semestres de formación

En el proceso de acreditación institucional del 2013 se midió el indicador "Postgrado en formación". La UPSE ha becado en los últimos 3 años al exterior a 25 docentes quienes se encuentran en diversas partes del mundo efectuando sus estudios de doctorado. Y maestrías en el ámbito nacional en el número de 90 , en diferentes áreas del conocimiento.

Entre agosto del 2012 y julio del 2013, fueron becados:

\begin{tabular}{|c|c|c|}
\hline \multicolumn{3}{|c|}{ DOCTORADOS = 4} \\
\hline Universidad & Programa & Origen \\
\hline $\begin{array}{l}\text { Universidad Es- } \\
\text { tatal de Belgo- } \\
\text { rod-Rusia (1) }\end{array}$ & $\begin{array}{l}\text { Doctorado en } \\
\text { Gerontología }\end{array}$ & Senescyt \\
\hline $\begin{array}{l}\text { Universidad de } \\
\text { Barcelona-Es- } \\
\text { paña (2) }\end{array}$ & $\begin{array}{l}\text { Doctorado en } \\
\text { Información y } \\
\text { Comunicación } \\
\text { en la Sociedad } \\
\text { del Conoci- } \\
\text { miento }\end{array}$ & Senescyt \\
\hline $\begin{array}{l}\text { Universidad } \\
\text { Deportiva Pe- } \\
\text { kín-China (1) }\end{array}$ & $\begin{array}{l}\text { Doctorado en } \\
\text { Gerencia De- } \\
\text { portiva }\end{array}$ & $\begin{array}{l}\text { Gobierno de la } \\
\text { República Po- } \\
\text { pular China con } \\
\text { el aval de la Se- } \\
\text { nescyt. }\end{array}$ \\
\hline
\end{tabular}

\begin{tabular}{|l|l|l|}
\multicolumn{3}{c}{ MAESTRÍAS = 2} \\
\hline Universidad & \multicolumn{1}{c|}{ Programa } & Origen \\
\hline $\begin{array}{l}\text { Australian Na- } \\
\text { tional Univer- } \\
\text { sity }\end{array}$ & $\begin{array}{l}\text { Maestría en Po- } \\
\text { líticas Públicas }\end{array}$ & Senescyt \\
\hline
\end{tabular}

\section{POST DOCTORADO=1}

\begin{tabular}{|c|c|c|}
\hline Universidad & Programa & Origen \\
\hline $\begin{array}{l}\text { Universidad } \\
\text { Deportiva Pe- } \\
\text { kín-China }\end{array}$ & $\begin{array}{l}\text { Post Doctora- } \\
\text { do Educación } \\
\text { Física y Entre- } \\
\text { namiento De- } \\
\text { portivo }\end{array}$ & $\begin{array}{l}\text { Gobierno de la } \\
\text { República Po- } \\
\text { pular China con } \\
\text { el aval de la Se- } \\
\text { nescyt. }\end{array}$ \\
\hline
\end{tabular}

Nuestra legión extranjera volvió a incrementarse en el presente año.

Del criterio Eficiencia Académica se destacan los indicadores siguientes:

\section{INDICADOR.- EFICIENCIA TERMINAL}

Las tasas alcanzadas en los años 2010 y 2012 en las cohortes de graduación fueron del $10 \%$ y $20,45 \%$ respectivamente, sobre una base de calidad del $60 \%$ de eficiencia terminal. Para incrementar la eficiencia terminal se han realizado y se están realizando planes de titularización de estudiantes de acuerdo a las correspondientes cohortes.

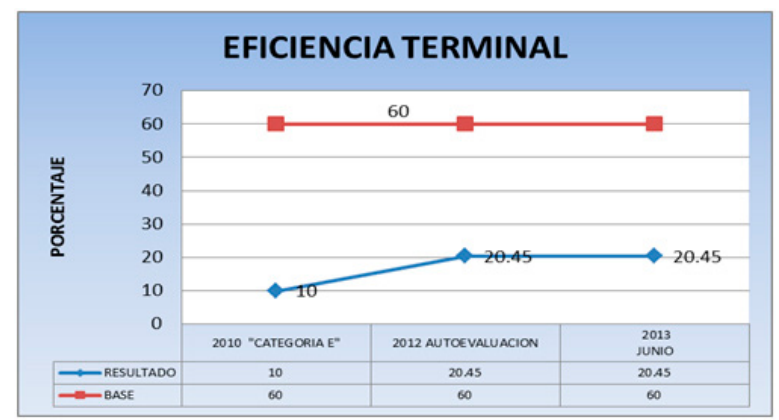

Del criterio Investigación destacamos los siguientes indicadores:

\section{INDICADOR: PLANIFICACIÓN DE LA INVESTI- GACIÓN}

El cumplimiento es total puesto que hoy existe un plan confeccionado y aplicado con rigurosidad.

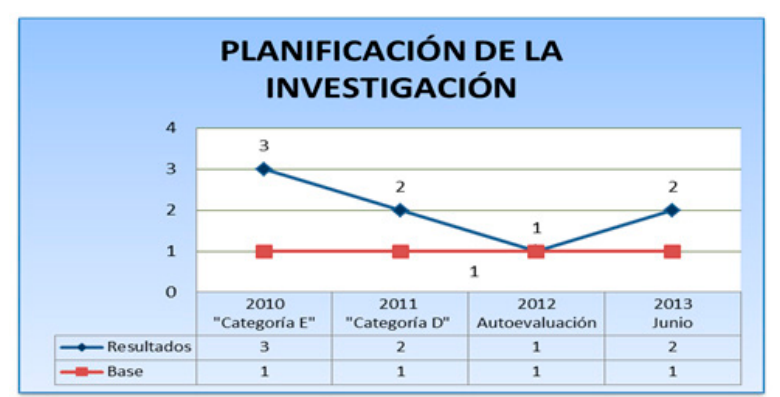

Del criterio Organización merecen destacarse los indicadores siguientes: 
INDICADOR: USO DEL SEGUIMIENTO A GRADUADOS

En el 2010 no se realizaba un seguimiento continuo a los graduados, actualmente se cuenta con un programa que tiene establecidos procedimientos claros para buscar la integración, participación y compromiso de nuestros graduados.

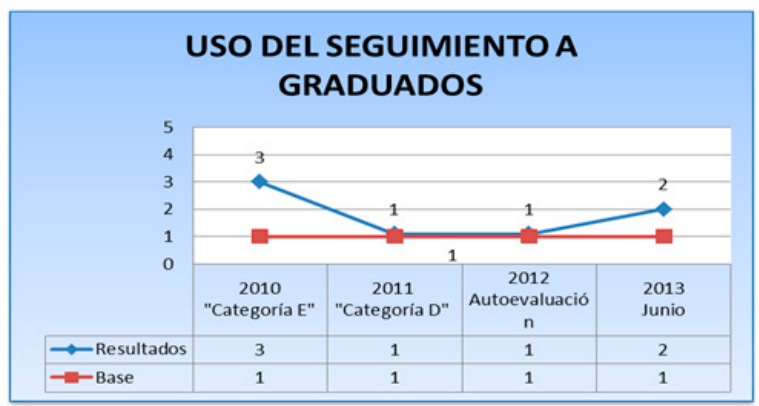

INDICADOR: PROGRAMAS DE VINCULACIÓN En el 2010 se ejecutaron únicamente actividades de vinculación con la colectividad, en la actualidad se cuenta con un departamento que trabaja en conjunto con las carreras para desarrollar programas y proyectos de vinculación. En el 2011, se tenían 10 programas y en el 2012 a junio del 2013 se cuenta con un total de 39 programas.

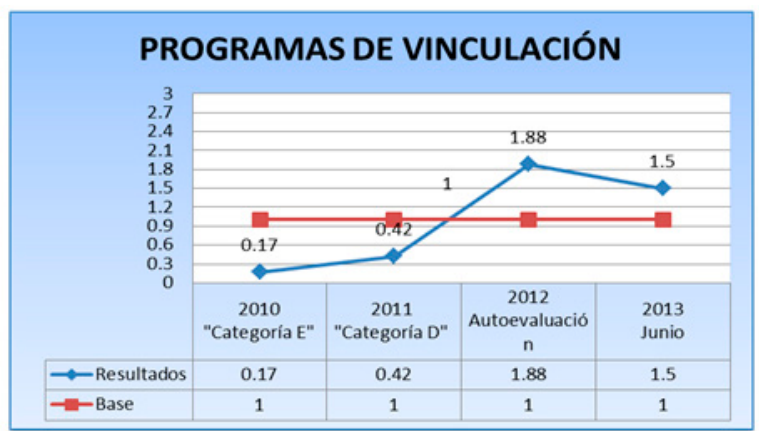

INDICADOR: RENDICIÓN ANUAL DE CUENTAS

En la actualidad, anualmente se rinde cuentas a la comunidad universitaria, a través de la sesión solemne de aniversario, publicaciones específicas impresas e información a través de los diferentes medios de comunicación.

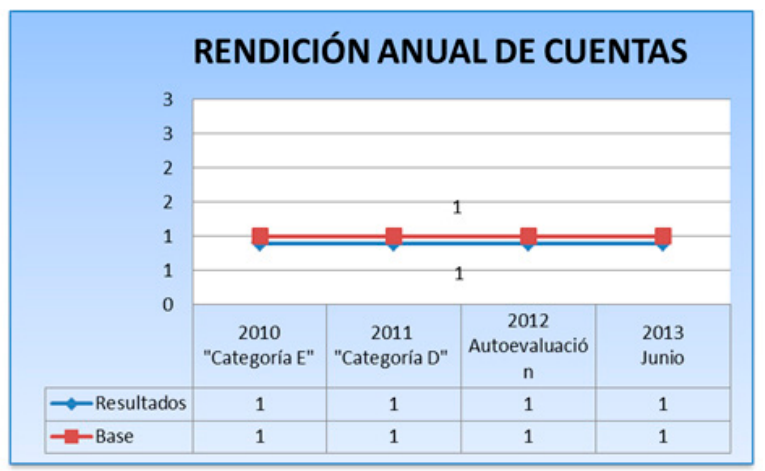

INDICADOR: TRANSPARENCIA EN LA INFORMACIÓN FINANCIERA

En el 2010 se consideró que la información sobre presupuestos y sus modificaciones no se remitían a los organismos competentes, ni se publicaban en la página web institucional. En la actualidad, se publica en la página web la información pertinente tanto académica como administrativa.

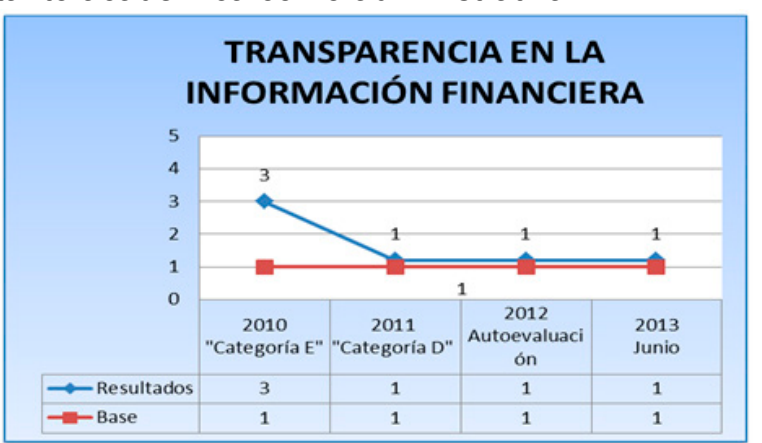

INDICADOR: ÉTICA Y RESPONSABILIDAD

En el 2010 y 2011 este indicador no se evaluó, pero la institución cuenta con organismos que regulan el comportamiento de la comunidad universitaria: Comisión Académica, Comisión Laboral y Comisión de Evaluación Interna. Y hoy, está integrada la Comisión de Ética cuyo Reglamento será próximamente reformado por el Consejo Superior.

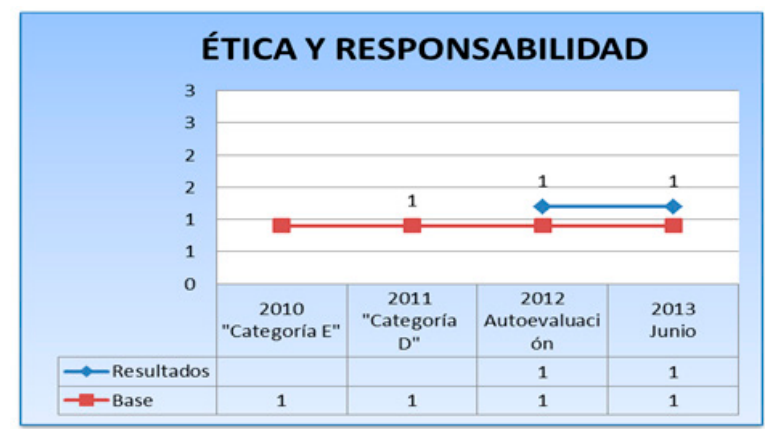

\section{INDICADOR: CALIDAD DEL GASTO}

Desde el 2010 hasta la presente fecha hemos trabajado en la racionalidad del gasto como lo muestra la gráfica. En el periodo académico 20122013 se incrementó un 10\% las remuneraciones de los docentes. Es la Academia hoy, el estamento de mayor inversión, puesto que es el pilar fundamental de la institución. En el año 2012 se ejecutó el $48 \%$ del presupuesto en remuneración docente y el $24 \%$ en remuneraciones administrativas. Este desequilibrio, que lo heredamos, está reducido notablemente. $Y$ aspiramos a continuar

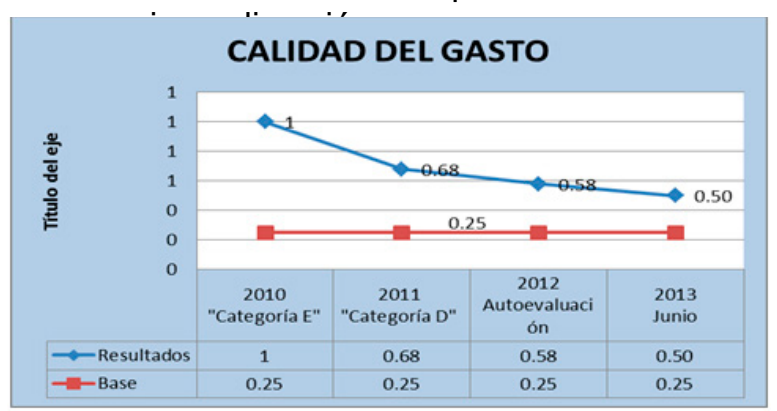


INDICADOR: ACCIÓN AFIRMATIVA

Desde el 2012 a junio del 2013 existen y se aplican las políticas de acción afirmativa.

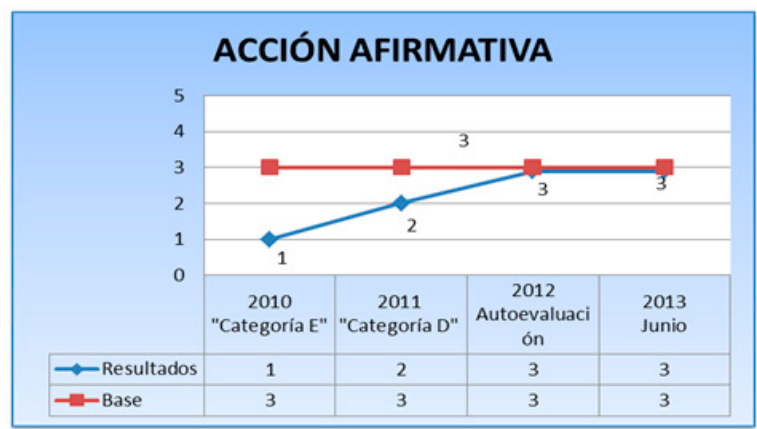

INDICADOR: INNOVACIÓN TECNOLÓGICA

En el 2010 no se contaba con todos los procesos automatizados, se tenía un cumplimiento parcial; actualmente se cuenta con diferentes módulos informáticos tales como: matriculación, registro y consulta de notas, blogs para docentes, control de syllabus, entre otros.

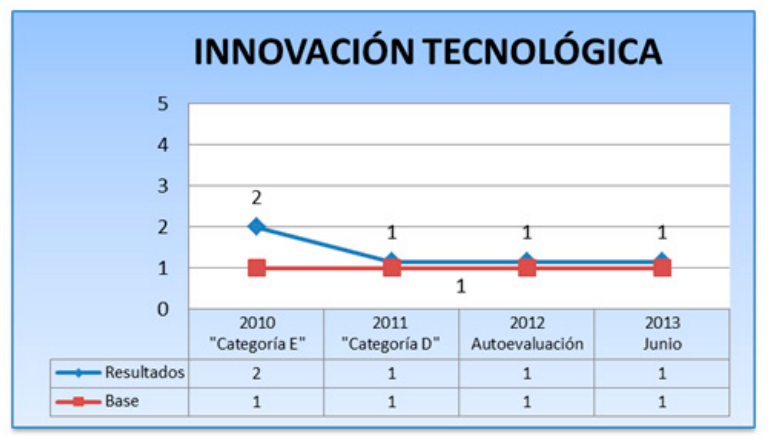

INDICADOR: COBERTURA A ESTUDIANTES

Del 2012 a junio del 2013 se han instalado infraestructuras para cobertura inalámbrica. Aún tenemos problemas, esperamos efectuar la inversión correspondiente para que la navegación sea total. Vamos avanzando.

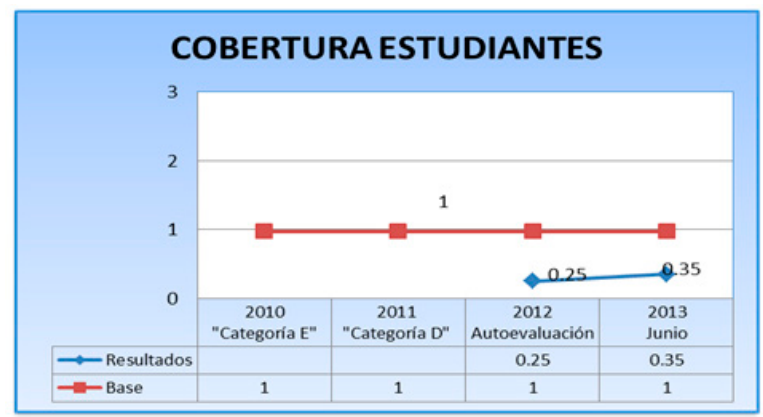

Al finalizar el proceso de autoevaluación registramos las fortalezas siguientes:

\section{FORTALEZAS}

-Planta docente especializada con título de cuarto nivel.

- Reglamentos y normativas claras, publicadas en la página web institucional y socializada con la comunidad universitaria.
- Espacios de Bienestar Universitario adecuados

- Innovación tecnológica que presta facilidades y fortalece los procesos académicos y administrativos

- Proceso automatizado de gestión de biblioteca

- Transparencia en la ejecución presupuestaria - Existencia de procesos de seguimiento a graduados, y planes de mejoras académicas diseñados a partir de los resultados.

- Programa de Seguimiento a Graduados consolidado que integran elementos tecnológicos para levantamiento de información y reuniones periódicas con graduados y autoridades con fines académicos.

- Programas de Vinculación con la colectividad estructurados en base a un protocolo estandarizado para el efecto.

- Plataforma informática que permite poner a disposición de estudiantes y docentes varios servicios en línea.

\section{5.- PLAN DE MEJORAS}

Como consecuencia de los resultados del proceso de autoevaluación se ha diseñado un nuevo Plan de Mejoras hasta diciembre del 2013, que contempla las siguientes metas:

\section{CRITERIO: ACADEMIA}

- Incrementar el porcentaje de docentes con maestría y Ph.D en un $70 \%$ y $5 \%$ respectivamente.

- Contar con un mínimo del $20 \%$ de docentes que estén cursando maestrías (que no tengan título de cuarto nivel) y $5 \%$ que estén cursando $\mathrm{PhD}$.

- Asignar la dedicación de tiempo completo al $100 \%$ de docentes con título de $\mathrm{PhD}$.

- Incrementar al 70\% de docentes a tiempo completo

- Mantener un promedio de 12 horas semanales de carga horaria de los docentes a tiempo completo durante el período académico vigente.

- Contar con el $70 \%$ de docentes con dedicación a tiempo completo

- Mantener un promedio de 10 horas semanales de carga horaria de los docentes a medio tiempo y tiempo parcial $100 \%$ de cumplimiento del plan de titularización

- El $80 \%$ de docentes titulares tendrán dedicación de tiempo completo

- $100 \%$ cumplimiento de la normativa

- Cumplimiento total del Reglamento de Escalafón y Carrera Docente.

- Cumplimiento total del Reglamento de Evaluación de Desempeño Docente vigente 
- Cumplimiento total de la escala remunerativa del escalafón

- $90 \%$ de los docentes con dedicación de tiempo completo con contrato anual

- $50 \%$ de participación docentes de género femenino en cargos de dirección académica.

- $50 \%$ docentes titulares género femenino

\section{CRITERIO: EFICIENCIA ACADÉMICA}

- $90 \%$ de los egresados de la cohorte 2008 graduados

- Máximo 10\% de deserción estudiantil

\section{CRITERIO: INVESTIGACIÓN}

- 1 Plan de Investigación

- 1 publicación anual de artículo científico por docente PhD o que esté cursando estudios de posgrado en el exterior

- Publicar 1 libro por Facultad

\section{CRITERIO: ORGANIZACIÓN}

- Reestructuración del número de las unidades académicas

- Cumplimiento total de los procesos de seguimiento a graduados

- 2 programas de vinculación con la colectividad por carrera

- $10 \%$ de programas y/o proyectos con presupuesto institucional asignado

- 1 informe anual de rendición de cuentas con 1000 ejemplares distribuidos

- Publicar al final del año la ejecución presupuestaria.

- Aplicar el manual de distribución de puestos y funciones según el estatuto reformado de la universidad

- Sistema de Información Gerencial en marcha

- Disponibilidad de la Información

- Actualización de la normativa Académica de conformidad a los reglamentos establecidos por el CES

- Documentar la aplicación de las políticas de acción afirmativa

\section{CRITERIO: INFRAESTRUCTURA}

- Establecer 15 estudiantes por cada sitio de trabajo en las bibliotecas.

- 8 títulos por Estudiante

- Implementar nuevas soluciones que permitan visualizar en la web, el acervo bibliográfico.

- 1 suscripción de bibliotecas virtuales para cada Facultad.

- $100 \%$ de conectividad en las áreas académicas

- Incrementar el ancho de banda por lo menos a 80 MBPS para acceso a internet

- Implementar nuevas soluciones informáticas que apoyen los procesos académicos

- $100 \%$ de edificios con cobertura inalámbrica
- $100 \%$ de las oficinas de docente TC. equipadas

- Un puesto de trabajo por cada dos docentes a medio tiempo

- Iniciar un plan de actualización tecnológica en las aulas.

- Asignar a cada aula un CPU para poder ofrecer las facilidades adecuadas para las actividades de enseñanza aprendizaje.

- Implementar áreas de reunión para los estudiantes con acceso wi-fi

\section{6.- CIERRE}

Tres son los grandes ejes que han impulsado el trabajo que venimos efectuando:

1. Forjar una visión de largo alcance, con una clara definición de nuestra misión y visión, donde se encuentra contenida nuestra identidad. Esto se ha ido logrando con la participación de todos los miembros de nuestra comunidad universitaria. Tenemos un proyecto en común que trasciende cualquier interés personal o inmediatista. Este proyecto, lo vamos concretando cada vez con más claridad y efectividad.

2. Mantener una comunicación fluida nos ha permitido alcanzar consensos y juntar voluntades. Alejando de nuestro entorno cualquier incomprensión o mal entendido. Esta vocación democrática es una de las herramientas más poderosas con la que cuenta nuestra universidad.

3. Por último, hemos aceptado de manera comprometida: exigencias y sacrificios, convencidos de que los cambios en la UPSE están provocando el cambio en la historia de la Provincia de Santa Elena.

"No se puede ser a la vez amado y temido, es mejor ser temido que amado". Dilema planteado por Nicolás Maquiavello; dilema que he experimentado a lo largo de este proceso.

Muchas decisiones, tomadas para enderezar el rumbo de nuestro accionar, no han sido populares. Pero... he tenido la satisfacción de comprobar que sus resultados han sido respetados y aquilatados posteriormente, por las consecuencias positivas obtenidas de su aplicación.

Tengo la firme convicción de que estamos haciendo historia.

Estamos cambiando visiones pesimistas que pesaban en nuestra vida institucional y que nos impedían transitar por la mejora continua. 
Estamos derrotando aquellas versiones que sostenían que la UPSE estaba condenada a ser una institución sin mayor significación.

Y en este caminar, hemos aprendido de instituciones amigas como la Escuela Politécnica del Litoral y de muchas otras IES ecuatorianas y extranjeras que se encuentran a tono con las exigencias de la sociedad del conocimiento y de este mundo globalizado.

En esta celebración, de los primeros quince años de nuestra joven universidad, volvemos a reiterar nuestro firme compromiso en la tarea de mantener a la UPSE en el corazón y en la confianza de todos los peninsulares.

MS.c Jimmy Candell Soto, Rector 